\title{
A Methodology on Work-flow Control System in Large-scaled job shop line
}

\author{
T. Kaihara ${ }^{1}$ and H. Imai ${ }^{2}$ \\ ${ }^{1}$ University of Marketing and Distribution Sciences \\ 3-1, Gakuen-nishi, Nishi, Kobe 651-21, JAPAN \\ Phone:+81-78-796-4402, Fax:+81-78-794-3054 \\ E-mail: kaihara@umds.ac.jp \\ ${ }^{2}$ Mitsubishi Electric Corporation \\ Manufacturing Engineering Center \\ 8-1, Tsukaguchi-Honmachi, Amagasaki, 661, JAPAN
}

\begin{abstract}
Work flow of very large-scaled job-shop type manufacturing systems is complicated due to a large number of processes and various types of work flow patterns. Sophisticated work flow management policy is required for efficient manufacturing operation, since the investment into such a large scaled manufacturing factory is considerably huge. A work flow control methodology which is based on future WIP(Work In Process) location estimation with virtual manufacturing model is proposed. The Architecture is simple and PC-based, and that facilitates the integration with conventional manufacturing system. The methodology has been proved to be efficient enough to apply into practical fields. Finally our concept on fully automated real time control system is presented.
\end{abstract}

Keywords

Schedulling, Simulation, Object-oriented, Manufacturing management system

\section{INTRODUCTION}

Work flow of very large-scaled job shop type manufacturing systems, such as semiconductor factories, is complicated due to a large number of processes and various types of work flow patterns. Sophisticated work flow management(or control) policy is required to attain highly performed manufacturing systems (Uzsoy, 1992)(Fuyuki, 1992). Conventionally work flow management is based on the knowledge of some skilled operators in manual handling manufacturing lines, although it is not proved optimum dispatching strategy. The manual operation based on the experience is required frequently even in the fully automated lines, since FA(Factory Automation) control system can't handle unexpected irregular occasions, such as long time machine faults. Additionally it is 
necessary in FA control system to reduce the manufacturing POP(Point of Production) data size for the response in time, despite the real time control generally collects the huge amount of the data. Since the investment cost into such a complicated manufacturing line is increasing gradually, it is essential to develop the work flow management system which manages to operate the manufacturing line efficiently (Yuki, 1996).

Virtual manufacturing model built in computers enables the previous performance evaluation and the decision makings about the factory planning, development, and management. The evaluation by the virtual model has been mainly used for planning phase or static evaluation of the factory, although the concept, that the model is applicable to dynamic factory operation, has been suggested by some research groups(Alting, 1988)(Wu, 1989).

The flexibility is one of the most important features in general software development. Hard-coded manufacturing control program, which requires much efforts and time for the modification, is not useful in practical use. Object-oriented programming paradigm facilitates efficient manufacturing system modelling(Kaihara, 1993a)(Kaihara, 1993b). Object-oriented programming is useful especially to human interface development in work flow control system, because that consists of many similar graphical windows. Prototype method leads to quicker development of the software.

\section{OBJECTIVES}

There are two types of manufacturing line in large-scaled job shop factory in terms of factory control automation level, fully automated and semi automated. Process machines and material handling facilities are controlled automatically in the former type, whereas the work flow control (schedulling and dispatching) are manually decided in the latter. Our research is mainly focused on the semi automated work flow control in this stage, since most factories adopt this type of control policy. The moderate cooperation between human experience and control logic is important and practical, because it is quite difficult to acquire expert's logic or instinct.

The major target of our research is the performance improvement of large-scaled job shop type manufacturing systems. Valuable information should be presented timely to operational staffs for higher decision makings by collecting, calculating, and filtering the factory data. Only present WIP(work in process) data is available in the conventional work flow control policy. The information of future WIP location helps greatly even experienced staffs to plan the appropriate schedulling or dispatching sequence into each resource. The manufacturing system performance is expected to be kept totally high, because the whole manufacturing system events should be included in the estimation. If something unexpected happens, such as machine failures or the increase of defect products, the higher decision makings with the virtual manufacturing model facilitate quick recovery.

Secondly the line operation efficiency on work flow management is expected. It is effective especially in semi automated operation factory, since it sometimes takes long time to negotiate to decide the WIP input order and such decision making is useful only temporarily. The WIP input sequence has been proved previously effective in the virtual manufacturing model, and few negotiations are required at the shop floor level. 


\section{CONCEPT AND SOFTWARE ARCHITECTURE}

\subsection{Concept}

The concept of our methodology is the interface agent based on the future WIP estimation by virtual manufacturing model with discrete-event simulation engine. Factory staffs can previously grasp the operational strategy to control manufacturing line appropriately, the amount of product output, lead time, and machine availability, and check if they meet the original production plan.

The future WIP location data is required at decision making occasions, perhaps several times in a day, in practical operation. Once the decision is fixed by the virtual manufacturing model, the work flow control sequence during the simulation scenario is obtained as the work tracking log data in the simulation results. In case the estimation results differ from actual factory performance, the comparison between simulation log and actual work flow enables to reveal the overestimated operational factors of the factory, because theoretically they are identical under correct factory parameters Proposed methodology is regarded as a leverage to exterminate hidden defects of the factory. Highly performed effective manufacturing system is attainable gradually through that feed back procedures.

\subsection{Structure}

Our prototype system based on the proposed methodology consists of four software modules, Work flow management module, Factory model module, Data translation module, and Automatic calculation module, shown in figure 1 . The work flow management module handles the work flow data and controls user I/F. It is developed with object-oriented programming paradigm in Visual Works language(Goldberg, 1986), so that it is easily applicable to other systems.

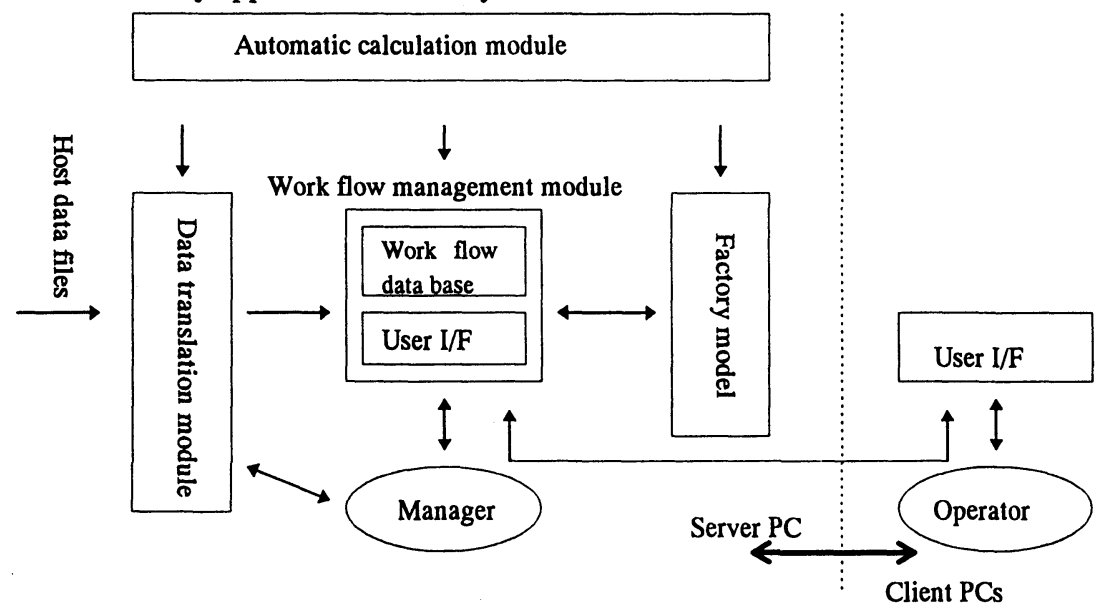

Figure 1 Software structure

General purpose virtual manufacturing modelling environment with discrete-event 
simulation engine is newly developed and applied into the factory model module in order to estimate the WIP location precisely. Template-based modelling method in which factory model is classified into two types, skeleton and specific models, is proposed and helps to facilitate efficient modelling. It is written in Microsoft Visual C- -+ +(Microsoft, 1996). This environment is applicable to factory planning and development as well as daily operation.

Manufacturing data files fetched from the upper main computer(Host computer) are translated and stored into the work flow data base automatically via the data translation module.

The automatic calculation module enables default decision making without the operator in actual use.

The major features of the system are as follows:

- Work flow management system with precise virtual manufacturing model

- EUC(End User Computing) environment based on MS Windows graphical user I/F

- OOP paradigm for software reusability

Figure 2. shows the system structure, which consists of Server PC(Windows NT) in management staff room and client PCs(Windows 3.1) in manufacturing shop floor.

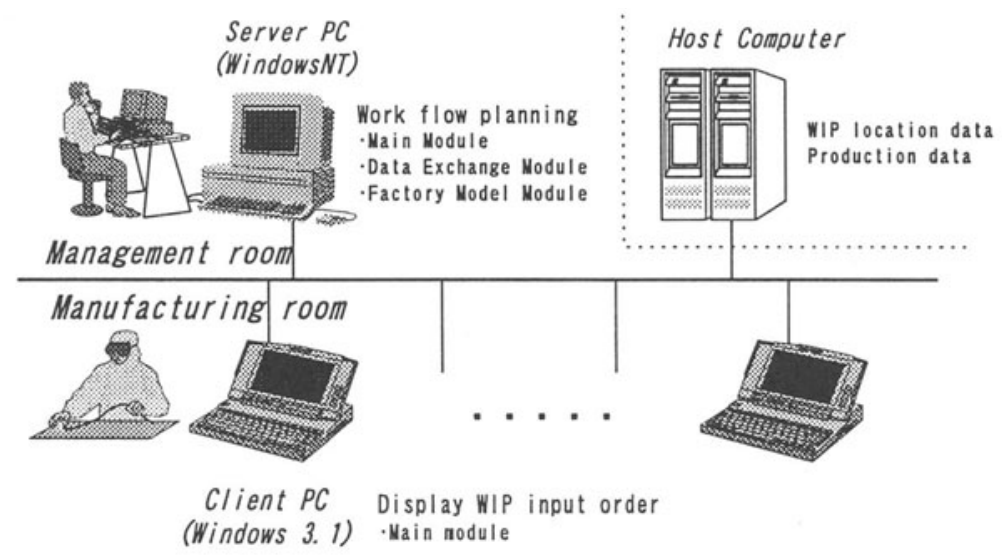

Figure 2 System structure

The host computer collects and maintains the manufacturing standard data and realtime information, such as process data, process flow, current WIP location and so forth. The server computer fetches several host data files for the WIP location and simulation via the data translation module. The work dispatching lists of each machines are created automatically from the work tracking log data in the simulation result, shown in figure 3. Some sophisticate dispatching rules which have been endorsed by static simulation are installed in the main module. In case some unexpected event happens, managers can try some simulation scenarios under new dispatching policy, compare the results with the original plan, and finally decide the appropriate strategy. The fixed dispatching order is transferred into each client PC to display the dispatching list to shop floor operators. The communication between the server PC and the client PCs is established by standard OOP 
protocol, CORBA. Object images produced in the server can be handled in each client transparently. After a product set into the process, operators are supposed to put a mark at the product on the display. The current manufacturing progress compared with the original plan is shown on the screen, then the manager can realize it anytime at a glance.

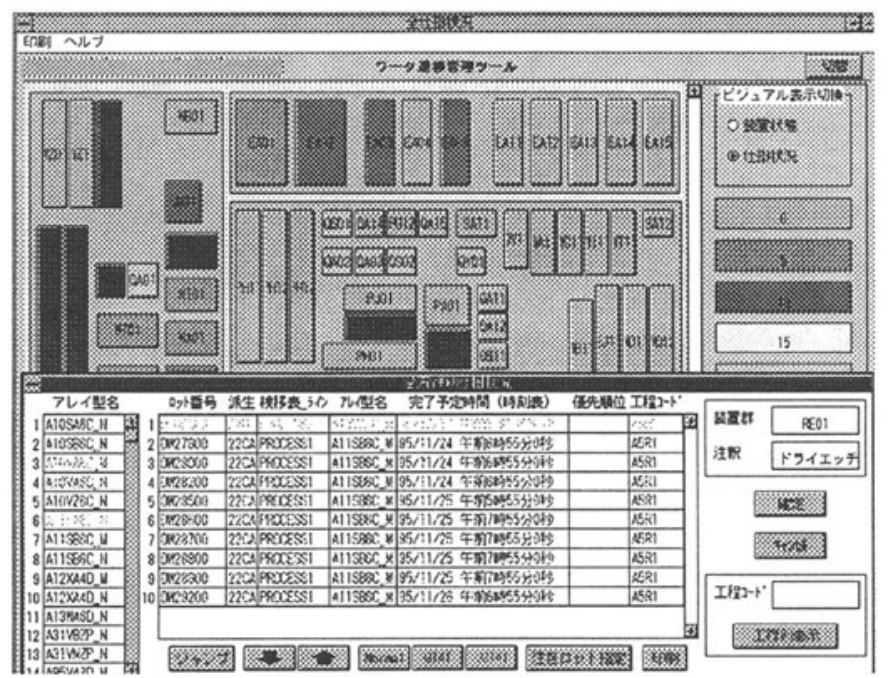

Figure 3 A Display example of User I/F(WIP layout map \& dispatching plan)

\section{CASE STUDY}

The prototype system has been installed into practical large-scaled job shop lines including about 60 process machines. Several satisfactory results were obtained:

1. Decrease in production lead time by $10 \%$

2. Reduction of the amount of shop floor operation by 10,000 hours/year

3. Easy installation and modification of the system

4. Significant reduction of the total investment cost into the PC-based work flow control system

\section{CONCLUSIONS}

Proposed work flow control methodology with virtual manufacturing model is quite simple and has been proved to be practical with human-computer interaction.

This methodology is also applicable in fully automated FA factories. Our opinion is that real time work flow control is not always suitable for large-scaled job shop manufacturing system due to complicated control algorithm and huge amount of data size to handle. The efficiency is not so obvious in spite of large investment. According to our methodology, the work flow control standard table is created automatically at each decision making point, perhaps several times in a day, and FA controller could just follow 
the control table for the time period. If the difference would happens between the location of virtual and actual works, the information could be feed backed and reset at the next decision making time. New research project is now in progress focused on FA factories.

\section{REFERENCES}

Alting, L.(1988) Extended applications of simulation in manufacturing system; Annals of the CIRP, Vol.37, pp.417-420

Fuyuki, M. and Inoue I.(1992) LISP-A Simulation System for Large-Scale-Production Planning Activity Support - Model and Simulator; Journal of the Japan Society for Simulation Technology; Vol.11, No.4, pp73-83

Goldgerg, A. and Robinsons D.(1986) Smalltalk80: The Language and its Implementation, Addison-Wesley

Kaihara, T. and Bcsant C.B.(1993a) Object-oriented flexiblc and integrated manufacturing system modelling; 1993 CompEuro Proceedings, pp.312-319

Kaihara, T. and Besant C.B.(1993b) Manufacturing System Modelling Structure based on Object Oriented Paradigm; The Proceedings of the 1993 Summer Simulation Conference, pp.831-836

Microsoft Corp.(1996) Microsoft Visual C++ Version 4.0 Development System for Windows 95 and Windows NT

Uzsoy R., Lee C. and Martin-Vega L.A.(1992) A Review of production planning and scheduling in the semiconductor industry; IIE Transactions, Vol.24, No.4, pp.47-60

Wu, S.D. and Wysk R.A.(1989) An application of discrete-event simulation to on-line control and scheduling in flexible manufacturing; Int. J. Production Research, Vol.27, No.9, pp.1603-1623

Yuki, H., Saito H., Imai H., and Kaihara T.(1996) Work-flow management system with virtual manufacturing model; Advances in Production Management Systems, pp55-58

\section{BIOGRAPHY}

Toshiya Kaihara received the B.E. and M.E. degrees in precision engineering from Kyoto University, Kyoto, Japan, in 1983 and 1985, respectively. He joined Mitsubishi Electric Corp. in 1985, where he worked at manufacturing system development. He received the $\mathrm{Ph}$. D. degree in mechanical engineering from Imperial College, University of London, London, UK, in 1994. He joined University of Marketing and Distribution Sciences in 1996.

$\mathrm{He}$ is a member of IEEE and Japan Society for Precision Engineering.

Hiroshi Imai received the B.E. degree in mechanical engineering from Tokyo University, Tokyo, Japan, in 1976. He joined Mitsubishi Electric Corp. in 1976, where he works at manufacturing system development. His current interests are PC-based FA control system using precise manufacturing simulator and real time $\mathrm{DB}$, and decision support system for shop floor operator. 